\title{
Bayesian Inference on the Shape Parameter and Future Observation of Exponentiated Family of Distributions
}

\author{
Sanku Dey ${ }^{1}$ and Sudhansu S. Maiti ${ }^{2}$ \\ ${ }^{1}$ Department of Statistics, St. Anthony's College, Shillong 793 001, India \\ ${ }^{2}$ Department of Statistics, Visva-Bharati University, Santiniketan 731 235, India \\ Correspondence should be addressed to Sudhansu S. Maiti, dssm1@rediffmail.com \\ Received 17 May 2011; Accepted 5 September 2011 \\ Academic Editor: Mohammad Fraiwan Al-Saleh \\ Copyright (C) 2011 S. Dey and S. S. Maiti. This is an open access article distributed under the \\ Creative Commons Attribution License, which permits unrestricted use, distribution, and \\ reproduction in any medium, provided the original work is properly cited. \\ The Bayes estimators of the shape parameter of exponentiated family of distributions have been \\ derived by considering extension of Jeffreys' noninformative as well as conjugate priors under \\ different scale-invariant loss functions, namely, weighted quadratic loss function, squared-log \\ error loss function and general entropy loss function. The risk functions of these estimators have \\ been studied. We have also considered the highest posterior density (HPD) intervals for the \\ parameter and the equal-tail and HPD prediction intervals for future observation. Finally, we \\ analyze one data set for illustration.
}

\section{Introduction}

Let $X$ be a random variable whose cumulative distribution function (cdf) and probability density function (pdf) are given by

$$
\begin{gathered}
G(x ; \alpha, \theta)=F^{\alpha}(x ; \theta), \\
g(x ; \alpha, \theta)=\alpha F^{\alpha-1}(x ; \theta) f(x ; \theta),
\end{gathered}
$$

respectively. Here $F(\cdot, \theta)$ is the continuous baseline distribution function with the corresponding probability density function $f(x ; \theta)$, and $\theta$ may be vector valued, and $\alpha$ is the positive shape parameter. Then, $X$ is said to be belonging to the exponentiated family of distributions (abbreviated as EFD) or the proportional reversed hazard family.

If the baseline distribution is exponential, then it is called the generalized exponential (GE) distribution in the literature. In recent years, an impressive array of papers has been 


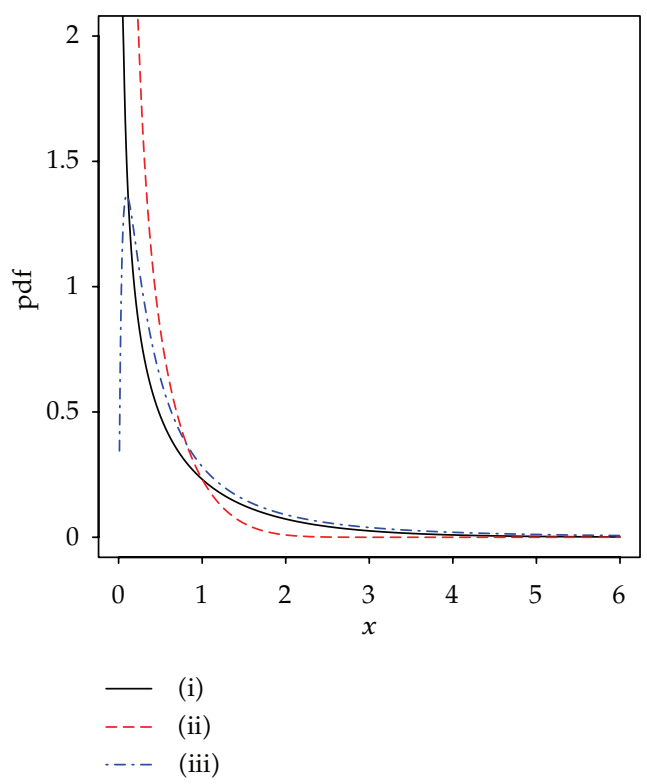

(a)

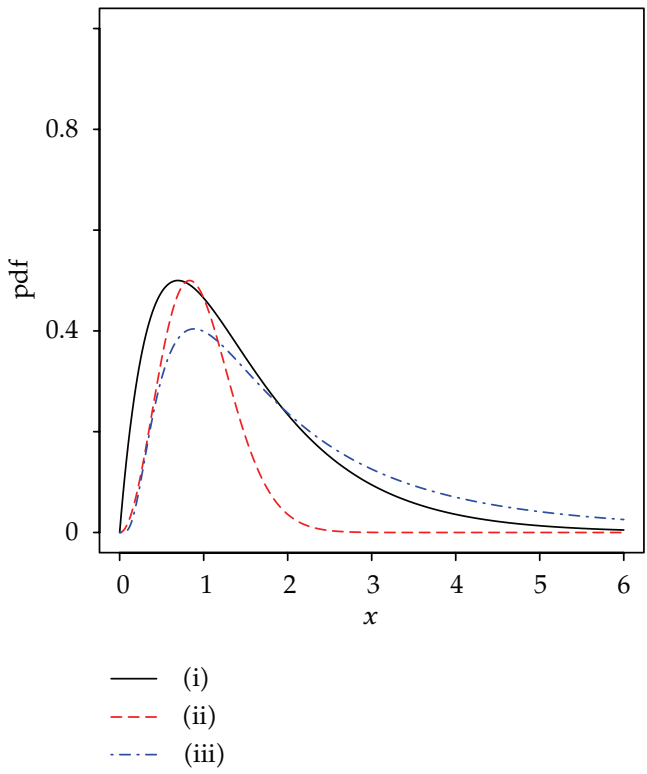

(b)

Figure 1: Exponentiated distributions for $\alpha=0.5$ and $\alpha=2$.

devoted to study the behavioral patterns of the parameters of the generalized exponential distribution using both classical and Bayesian framework, and a very good summary of this work can be found in Gupta and Kundu [1-4], Raqab [5], Raqab and Ahsanullah [6], Zheng [7], Raqab and Madi [8], Alamm et al. [9], Singh et al. [10], Dey [11], and the references cited there for some recent developments on GE distribution. If the baseline distribution is Weibull, then it is called the exponentiated Weibull distribution. Mudholkar and Srivastava [12], Nassar and Eissa [13], and Singh et al. [14] have studied this distribution.

In this paper, we assume that $F(x, \theta)=F(x)$ is known, but the shape parameter $\alpha$ is unknown. Then, the cdf and pdf become

$$
\begin{gathered}
G(x ; \alpha)=F^{\alpha}(x), \\
g(x ; \alpha)=\alpha F^{\alpha-1}(x) f(x),
\end{gathered}
$$

respectively. If $F(x)$ is symmetric, then $G(x ; \alpha)$ will be skewed distribution for different values of $\alpha(\neq 1)$. Hence $\alpha$ can be considered as a skewness parameter. Gupta and Gupta [15] have shown that positively skewed data can be analyzed very well for normal baseline distribution. Again $\alpha$ is the parameter of the proportional reversed hazard model in lifetime data analysis. For its various important roles, we are interested to find out the Bayes estimators and their performances under different loss functions using different priors. In Figure 1, the shape of (i) exponentiated distribution with $F(x)=1-e^{-x}$, (ii) exponentiated Rayleigh, distribution with $F(x)=1-e^{-x^{2}}$, and (iii) exponentiated lognormal distribution with $F(x)=\Phi(\ln x)$ has been shown for $\alpha=0.5$ and $\alpha=2$, respectively.

The paper is categorized into the following sections. Section 2 has a brief description of the prior distributions and loss functions. The Bayes estimators and associated risk functions 
are provided in Sections 3 and 4, respectively. Section 5 presents the highest posterior density (HPD) interval for $\alpha$. Section 6 is devoted to finding out the predictive distributions and equal-tail Bayesian predictive interval for the future observation. Section 7 deals with the Bayes predictive estimator and HPD prediction interval for a future observation. Section 8 presents the data application based on a real life data set. The paper ends with a concluding remark in Section 9.

\section{Prior and Loss Functions}

The Bayesian inference requires appropriate choice of prior(s) for the parameter(s). From the Bayesian viewpoint, there is no clear cut way from which one can conclude that one prior is better than the other. Nevertheless, very often priors are chosen according to one's subjective knowledge and beliefs. However, if one has adequate information about the parameter(s), it is better to choose informative prior(s); otherwise, it is preferable to use noninformative prior(s). In this paper we consider both type of priors: the extended Jeffreys' prior and the natural conjugate prior.

The extended Jeffreys' prior proposed by Al-Kutubi [16] is given as

$$
\pi_{2}(\alpha) \propto \frac{1}{\alpha^{2 c_{1}}}, \quad \alpha>0, c_{1}>0
$$

The conjugate prior in this case will be the gamma prior, and the probability density function is taken as

$$
\pi_{1}(\alpha)=\frac{b^{a}}{\Gamma(a)} \alpha^{a-1} e^{-b \alpha}, \quad \alpha, a, b>0
$$

With the above priors, we use three different loss functions for the model (1.1).

(1) The first loss function considered is called weighted quadratic loss function and is given by

$$
L_{1}(\alpha, \delta)=\left(\frac{\alpha-\delta}{\alpha}\right)^{2}
$$

where $\delta$ is a decision rule to estimate $\alpha$. $\delta$ is to be chosen such that

$$
\int_{0}^{\infty}\left(\frac{\alpha-\delta}{\alpha}\right)^{2} \pi(\alpha \mid \underline{x}) d \alpha
$$

is minimum. This can be equivalently written as

$$
\int_{0}^{\infty}(\alpha-\delta)^{2} q(\alpha \mid \underline{x}) d \alpha, \quad \text { with } q(\alpha \mid \underline{x})=\frac{\left(1 / \alpha^{2}\right) \pi(\alpha \mid \underline{x})}{\int_{0}^{\infty}\left(1 / \alpha^{2}\right) \pi(\alpha \mid \underline{x}) d \alpha}
$$


being minimum. Hence

$$
\tilde{\alpha}_{\mathrm{bq}}=\delta=E_{q}(\alpha \mid \underline{x})
$$

(2) The second one is the squared-log error loss function proposed by Brown [17] and is defined as

$$
L_{2}(\alpha, \delta)=(\ln \delta-\ln \alpha)^{2}=\left(\ln \frac{\delta}{\alpha}\right)^{2}
$$

This loss function is balanced with $\lim L_{2}(\alpha, \delta) \rightarrow \infty$ as $\delta \rightarrow 0$ or $\infty$. A balanced loss function takes both error of estimation and goodness of fit into account, but the unbalanced loss function only considers error of estimation. This loss function is convex for $\delta / \alpha \leq e$ and concave otherwise, but its risk function has a unique minimum with respect to $\delta$. The Bayes estimator for the parameter $\alpha$ under squared-log error loss function may be given as

$$
\tilde{\alpha}_{\mathrm{bsl}}=\exp [E(\ln \alpha \mid \underline{x})]
$$

where $E(\cdot)$ denotes the posterior expectation.

(3) The third loss function is a particular type of asymmetric loss functions called the general entropy loss function proposed by Calabria and Pulcini [18] (Podder and Roy [19] called it the modified linear exponential (MLINEX) loss function) and is given by

$$
L_{3}(\alpha, \delta)=w\left[\left(\frac{\delta}{\alpha}\right)^{\gamma}-\gamma \ln \left(\frac{\delta}{\alpha}\right)-1\right], \quad \gamma \neq 0, w>0 .
$$

If we replace $\delta-\alpha$ in place of $\ln (\delta / \alpha)$, that is, $\ln \delta-\ln \alpha$, we get the linear exponential (LINEX) loss function, $w\left[e^{\gamma(\delta-\alpha)}-\gamma(\delta-\alpha)-1\right]$. Without loss of generality, we assume that $w=1$. If $\gamma=1$, it is the entropy loss function. Under this general entropy loss function, the Bayes estimator of $\alpha$ is obtained as follows:

$$
\tilde{\alpha}_{\text {bge }}=\left[E_{\alpha}\left(\alpha^{-\gamma}\right)\right]^{-1 / \gamma} \text {. }
$$

\section{Estimation of Parameter}

Let us consider a random sample $\underline{x}=\left(x_{1}, x_{2}, \ldots, x_{n}\right)$ of size $n$ from the exponentiated family of distributions. Then the likelihood function of $\alpha$ for the given sample observation is

$$
\begin{aligned}
L(\alpha \mid \underline{x}) & =\alpha^{n} \prod_{i=1}^{n} F^{\alpha-1}\left(x_{i}\right) f\left(x_{i}\right) \\
& =\alpha^{n} e^{\alpha \sum_{i=1}^{n} \ln F\left(x_{i}\right)} \prod_{i=1}^{n} \frac{f\left(x_{i}\right)}{F\left(x_{i}\right)} .
\end{aligned}
$$

Here, the maximum likelihood estimator (MLE) of $\alpha$ is $\widehat{\alpha}_{\text {mle }}=n / T$, with $T=-\sum_{i=1}^{n} \ln F\left(x_{i}\right)$. 


\subsection{Estimation under the Assumption of Extended Jeffreys' Prior}

Combining the prior distribution in (2.1) and the likelihood function, the posterior density of $\alpha$ is derived as follows:

$$
\pi_{1}(\alpha \mid \underline{x})=\frac{T^{n-2 c_{1}+1}}{\Gamma\left(n-2 c_{1}+1\right)} e^{-\alpha T} \alpha^{n-2 c_{1}}, \quad \alpha>0,
$$

which is a gamma distribution $G\left(n-2 c_{1}+1, T\right)$.

For differnt derivations in this section and subsequent sections, we use the expressions $\Gamma(p)=\int_{0}^{\infty} x^{p-1} e^{-x} d x, \Gamma^{\prime}(p)=\int_{0}^{\infty} \ln x x^{p-1} e^{-x} d x, \Gamma^{\prime \prime}(p)=\int_{0}^{\infty}(\ln x)^{2} x^{p-1} e^{-x} d x, \psi(p)=d \ln \Gamma(p) /$ $d p=\Gamma^{\prime}(p) / \Gamma(p)$, the digamma function, and $\psi^{\prime}(p)=d^{2} \ln \Gamma(p) / d p^{2}=\left(\left(\Gamma^{\prime \prime}(p) \cdot \Gamma(p)-\left[\Gamma^{\prime}(p)\right]^{2}\right) /\right.$ $\left.\Gamma^{2}(p)\right)$, the trigamma function.

Using extended Jeffreys' prior of the form (2.1), the Bayes estimators of $\alpha$ under weighted quadratic, squared-log error and general entropy loss functions are derived as follows:

$$
\begin{gathered}
\tilde{\alpha}_{\mathrm{bq}}^{E}=\frac{n-2 c_{1}-1}{T}, \\
\tilde{\alpha}_{\mathrm{bsl}}^{E}=\frac{e^{\psi\left(n-2 c_{1}+1\right)}}{T}, \\
\tilde{\alpha}_{\mathrm{bge}}^{E}=\left[\frac{\Gamma\left(n-2 c_{1}+1\right)}{\Gamma\left(n-2 c_{1}+1-\gamma\right)}\right]^{1 / \gamma} \cdot \frac{1}{T}=\frac{k}{T},
\end{gathered}
$$

with $k=\left[\Gamma\left(n-2 c_{1}+1\right) / \Gamma\left(n-2 c_{1}+1-\gamma\right)\right]^{1 / \gamma}$, respectively.

Remark 3.1. We get the Jeffreys' noninformative prior for $c_{1}=1 / 2$ and the Hartigan's noninformative prior for $c_{1}=3 / 2$.

\subsection{Estimation under the Assumption of Conjugate Prior}

Combining the prior distribution in (2.2) and the likelihood function, the posterior density of $\alpha$ is derived as follows:

$$
\pi_{2}(\alpha \mid \underline{x})=\frac{T_{b}^{n+a}}{\Gamma(n+a)} e^{-\alpha T_{b}} \alpha^{n+a-1}, \quad \alpha>0,
$$

which is a gamma distribution $G\left(n+a, T_{b}\right)$ with $T_{b}=T+b$.

Using a conjugate prior of the form (2.2), the Bayes estimators under weighted quadratic, squared-log error and general entropy loss functions are derived as follows:

$$
\begin{gathered}
\tilde{\alpha}_{\mathrm{bq}}^{c}=\frac{n-2+a}{T_{b}}, \\
\tilde{\alpha}_{\mathrm{bsl}}^{c}=\frac{e^{\psi(n+a)}}{T_{b}}, \\
\tilde{\alpha}_{\mathrm{bge}}^{c}=\left[\frac{\Gamma(n+a)}{\Gamma(n+a-\gamma)}\right]^{1 / \gamma} \cdot \frac{1}{T_{b}},
\end{gathered}
$$


respectively. It is to be noted that Bayes' estimators given in (3.5), (3.6), and (3.7) depend on $a$ and $b$ which are the parameters of the prior distribution of $\alpha$. These parameters, that is, $a$ and $b$ could be estimated by means of empirical Bayes' procedure (see Lindley [20] and Awad and Gharraf [21]). Given the random sample $\underline{x}=\left(x_{1}, x_{2}, \ldots, x_{n}\right)$, the likelihood function of $\alpha$ is gamma density with parameter $(n+1, T)$. Hence it is proposed to estimate the prior parameters $a$ and $b$ from the sample by $n+1$ and $T$, respectively. Therefore, (3.5), (3.6), and (3.7) will become

$$
\begin{gathered}
\tilde{\alpha}_{\mathrm{bq}}^{c}=\frac{2 n-1}{2 T}, \\
\tilde{\alpha}_{\mathrm{bsl}}^{c}=\frac{e^{\psi(2 n+1)}}{2 T}, \\
\tilde{\alpha}_{\mathrm{bge}}^{c}=\left[\frac{\Gamma(2 n+1)}{\Gamma(2 n+1-\gamma)}\right]^{1 / \gamma} \cdot \frac{1}{2 T}=\frac{K_{1}}{2 T}, \quad \text { where } K_{1}=\left[\frac{\Gamma(2 n+1)}{\Gamma(2 n+1-\gamma)}\right]^{1 / \gamma},
\end{gathered}
$$

respectively.

\section{Risks of the Bayes Estimators}

Since $X$ follows the exponentiated family of distributions with parameter $\alpha$, then $T=$ $-\sum_{i=1}^{n} \ln F\left(x_{i}\right)$ is distributed as $G(n, \alpha)$. Then the probability density function of $T$ is

$$
h_{T}(t)=\frac{\alpha^{n}}{\Gamma(n)} e^{-\alpha t} t^{n-1}, \quad t>0
$$

Therefore,

$$
E_{h}\left(T^{-\gamma}\right)=\int_{0}^{\infty} t^{-\gamma} h_{T}(t) d t=\frac{\alpha^{n}}{\Gamma(n)} \int_{0}^{\infty} e^{-\alpha t} t^{n-\gamma-1} d t=\frac{\Gamma(n-\gamma)}{\Gamma(n)} \alpha^{\gamma}
$$

The risk function of $\tilde{\alpha}_{\mathrm{bq}}^{E}$ is

$$
\begin{aligned}
R\left(\tilde{\alpha}_{\mathrm{bq}}^{E}\right) & =E_{h}\left[L\left(\tilde{\alpha}_{\mathrm{bq}}^{E}, \delta\right)\right] \\
& =\frac{1}{\alpha^{2}}\left[\alpha^{2}-2 \alpha\left(n-2 c_{1}-1\right) E_{h}\left(\frac{1}{T}\right)+\left(n-2 c_{1}-1\right)^{2} E_{h}\left(\frac{1}{T^{2}}\right)\right] \\
& =\frac{1}{\alpha^{2}}\left[\alpha^{2}-2 \alpha\left(n-2 c_{1}-1\right) \frac{\alpha}{n-1}+\left(n-2 c_{1}-1\right)^{2} \frac{\alpha^{2}}{(n-1)(n-2)}\right] \\
& =\left[1-\frac{2\left(n-2 c_{1}-1\right)}{n-1}+\frac{\left(n-2 c_{1}-1\right)^{2}}{(n-1)(n-2)}\right] .
\end{aligned}
$$


Similarly, the risk functions of $\tilde{\alpha}_{\text {bsl }}^{E}$ and $\tilde{\alpha}_{\text {bge }}^{E}$ under squared-log error loss and general entropy loss functions are

$$
\begin{aligned}
& R\left(\tilde{\alpha}_{\text {bsl }}^{E}\right)=\psi^{\prime}(n)+\left[\psi\left(n-2 c_{1}+1\right)-\psi(n)\right]^{2}, \\
& R\left(\tilde{\alpha}_{\text {bge }}^{E}\right)=k^{\gamma} \frac{\Gamma(n-\gamma)}{\Gamma(n)}+\gamma[\psi(n)-\ln k]-1,
\end{aligned}
$$

respectively. The risk functions of $\widetilde{\alpha}_{\mathrm{bq}}^{c}, \widetilde{\alpha}_{\mathrm{bsl}}^{c}$, and $\widetilde{\alpha}_{\mathrm{bge}}^{c}$, assuming conjugate prior are

$$
\begin{gathered}
R\left(\tilde{\alpha}_{\mathrm{bq}}^{c}\right)=\left[1-\frac{2 n-1}{n-1}+\frac{(2 n-1)^{2}}{4(n-1)(n-2)}\right], \\
R\left(\widetilde{\alpha}_{\mathrm{bsl}}^{c}\right)=\psi^{\prime}(n)+[\psi(2 n+1)-\psi(n)-\ln 2]^{2}, \\
R\left(\tilde{\alpha}_{\mathrm{bge}}^{c}\right)=k_{1}^{\gamma} \frac{\Gamma(n-\gamma)}{\Gamma(n)}+\gamma\left[\psi(n)-\ln k_{1}+\ln 2\right]-1,
\end{gathered}
$$

respectively.

The risk functions of $\widehat{\alpha}_{\text {mle }}$ under weighted quadratic, squared-log error and general entropy loss functions are

$$
\begin{gathered}
R_{q}\left(\widehat{\alpha}_{\text {mle }}\right)=\left[1-\frac{2 n}{n-1}+\frac{n^{2}}{(n-1)(n-2)}\right], \\
R_{s l}\left(\widehat{\alpha}_{\text {mle }}\right)=\psi^{\prime}(n)+[\psi(n)-\ln n]^{2}, \\
R_{g e}\left(\widehat{\alpha}_{\text {mle }}\right)=n^{\gamma} \frac{\Gamma(n-\gamma)}{\Gamma(n)}+\gamma[\psi(n)-\ln n]-1,
\end{gathered}
$$

respectively.

The estimators, developed in Section 3, are studied here on the basis of their risks obtained under three different loss functions, namely, (a) weighted quadratic loss, (b) squared-log error loss, (c) general entropy loss for $\gamma=0.5$, (d) general entropy loss for $\gamma=1$, and (e) general entropy loss for $\gamma=1.5$. Risk functions of the proposed estimators are shown in Figures 2-5. The thick lines in each figure show the risk of the Bayes estimators under extended Jeffreys' prior and conjugate prior, and dotted lines show the risk of MLE under different loss functions. In Figure 2, risk functions have been plotted for all the loss functions under extended Jeffreys' prior for different values of $c_{1}$ and for $n=30$. It is observed that risks are increasing with the increase in $c_{1}$. Risks under general entropy loss for $\gamma<1$ are ordinarily less than those of weighted quadratic and squared-log error losses. For small values of $c_{1}$, risks of Bayes estimators are lower than those of maximum likelihood estimators for each loss function considered. The Bayes estimators perform better for some values of $c_{1}$ and loss functions under consideration; for example, in the figure, risk of $\tilde{\alpha}_{\mathrm{bq}}^{E}$ is less for $c_{1}<1.5$ (approximately) whereas that of $\widetilde{\alpha}_{\text {bsl }}^{E}$ is less for $c_{1}<0.8$ (approximately). Figures 3 and 4 show the risks for different values of $n$ for $c_{1}=0.5$ and 2, respectively. We find that the risks are decreasing with the increase in $n$ for all values of $c_{1}$. 


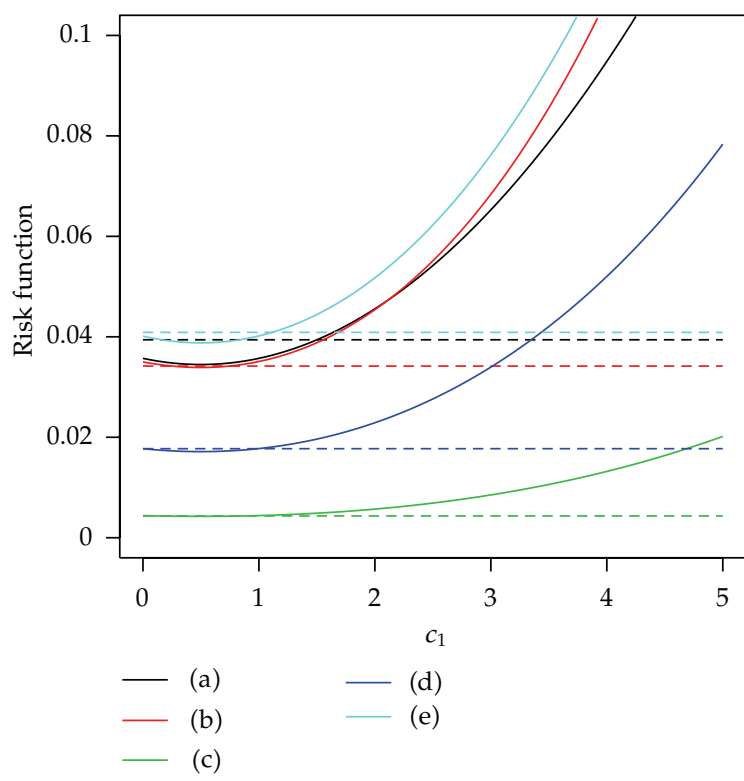

Figure 2: Risk function of estimators under the extended Jeffreys' prior for different values of $c_{1}$ with $n=30$.
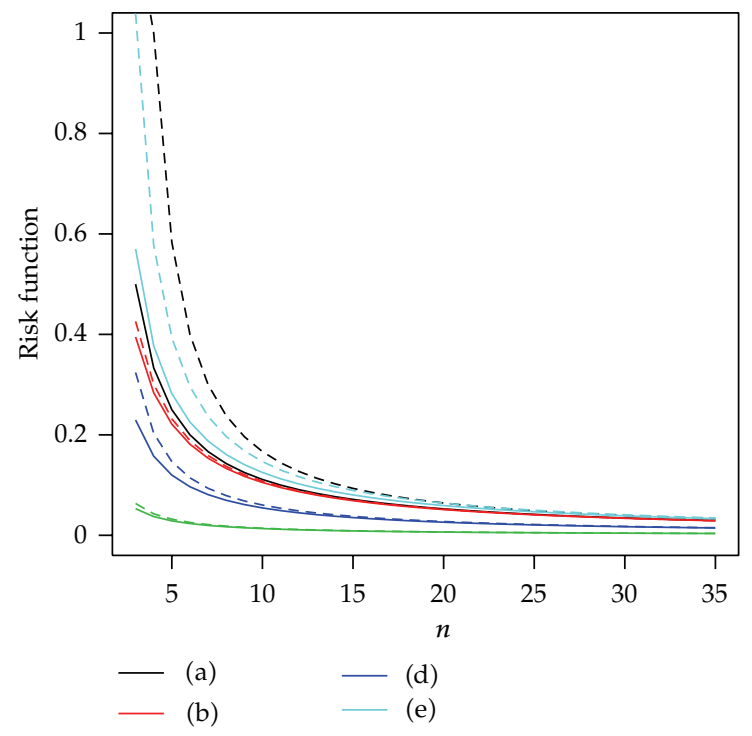

(c)

Figure 3: Risk function of estimators under the extended Jeffreys' prior for different values of $n$ with $c_{1}=$ 0.5 .

When we consider conjugate prior, we see that risks are less for the squared-log error and weighted quadratic losses than the general entropy loss, and only for these two losses, the Bayes estimators are less than the MLE for small $n$ (Figure 5). The risks under conjugate prior are generally higher than those under Jeffreys' prior. 

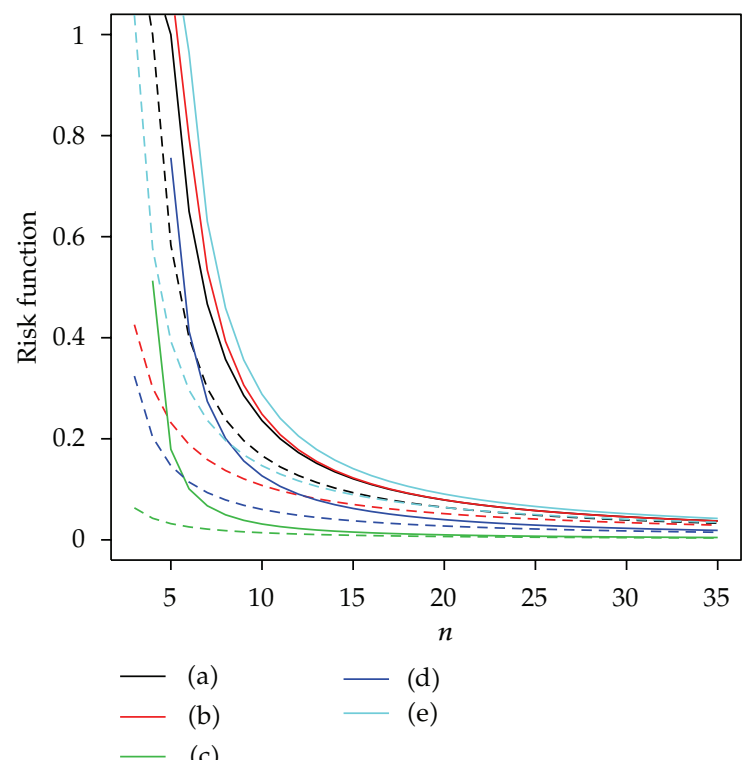

Figure 4: Risk function of estimators under the extended Jeffreys' prior for different $n$ with $c_{1}=2$.
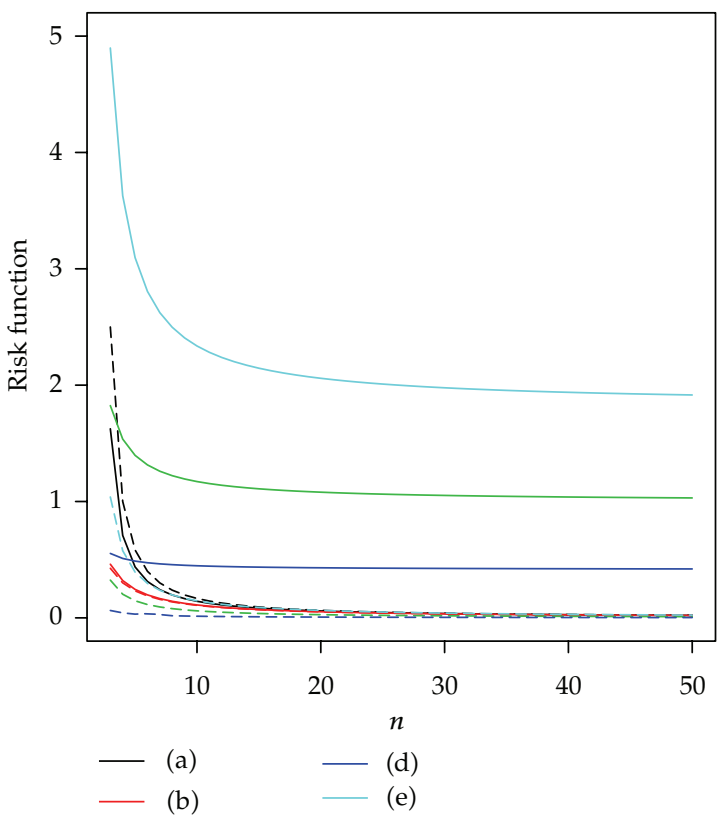

(c)

Figure 5: Risk function of estimators under conjugate prior for different $n$.

\section{Highest Posterior Density Intervals for $\alpha$}

In this section our objective is to provide a highest posterior density (HPD) interval for the unknown parameter $\alpha$ of the model (1.2). HPD interval is one of the most useful tools to measure posterior uncertainty. This interval is such that it includes more probable values 
of the parameter and excludes the less probable ones. Since the posterior density (3.2) is unimodal, the $100(1-\eta) \%$ HPD interval $\left[H_{1}^{E}, H_{2}^{E}\right]$ for $\alpha$ must satisfy

$$
\int_{H_{1}^{E}}^{H_{2}^{E}} \pi_{1}(\alpha \mid \underline{x}) d \alpha=1-\eta
$$

that is

$$
\begin{gathered}
\Gamma\left(n-2 c_{1}+1, H_{2}^{E} T\right)-\Gamma\left(n-2 c_{1}+1, H_{1}^{E} T\right)=1-\eta, \\
\pi_{1}\left(H_{1}^{E} \mid x\right)=\pi_{1}\left(H_{2}^{E} \mid x\right)
\end{gathered}
$$

that is

$$
\left[\frac{H_{1}^{E}}{H_{2}^{E}}\right]^{n-2 c_{1}}=e^{T\left(H_{1}^{E}-H_{2}^{E}\right)},
$$

simultaneously. The HPD interval $\left[H_{1}^{E}, H_{2}^{E}\right]$ is the simultaneous solution of (5.2) and (5.4).

Similarly, the posterior density (3.4) is unimodal, and the 100(1- $\eta) \%$ HPD interval $\left[H_{1}^{c}, H_{2}^{c}\right]$ for $\alpha$ must satisfy

$$
\int_{H_{1}^{c}}^{H_{2}^{c}} \pi_{2}\left(\alpha \mid T_{a}\right) d \alpha=1-\eta
$$

that is

$$
\begin{gathered}
\Gamma\left(n+b, H_{2}^{c} T_{a}\right)-\Gamma\left(n+b, H_{1}^{c} T_{a}\right)=1-\eta, \\
\pi_{2}\left(H_{1}^{c} \mid T_{a}\right)=\pi_{2}\left(H_{2}^{c} \mid T_{a}\right),
\end{gathered}
$$

that is

$$
\left[\frac{H_{1}^{c}}{H_{2}^{c}}\right]^{n+b-1}=e^{T_{a}\left(H_{1}^{c}-H_{2}^{c}\right)},
$$

simultaneously. Therefore, the HPD interval $\left[H_{1}^{c}, H_{2}^{c}\right]$ is the simultaneous solution of (5.6) and (5.8). If $a$ and $b$ are not known, then substituting the empirical Bayes estimate of $a$ and $b$, we get the equations as follows:

$$
\begin{gathered}
\Gamma\left(2 n+1,2 H_{2}^{c} T\right)-\Gamma\left(2 n+1,2 H_{1}^{c} T\right)=1-\eta, \\
{\left[\frac{H_{1}^{c}}{H_{2}^{c}}\right]^{2 n}=e^{2 T\left(H_{1}^{c}-H_{2}^{c}\right)},}
\end{gathered}
$$

respectively. 


\section{Predictive Distribution}

In this section our objective is to obtain the posterior predictive density of future observation, based on current observations. Another objective is to attain equal-tail Bayesian prediction interval for the future observation and then compare this interval with frequentist predictive interval. The posterior predictive distribution for $y=x_{n+1}$ given $\underline{x}=\left(x_{1}, x_{2}, \ldots, x_{n}\right)$ under (3.2) is defined by

$$
\begin{aligned}
\xi^{E}(y \mid \underline{x}) & =\int_{0}^{\infty} \pi_{1}(\alpha \mid \underline{x}) g(y ; \alpha) d \alpha \\
& =\frac{n-2 c_{1}+1}{T} \frac{1}{[1-(\ln F(y) / T)]^{n-2 c_{1}+2}} \frac{f(y)}{F(y)} .
\end{aligned}
$$

A $100(1-\eta) \%$ equal-tail prediction interval $\left[y_{1}^{E}, y_{2}^{E}\right]$ is the solution of

$$
\int_{0}^{y_{1}^{E}} \xi^{E}(y \mid \underline{x}) d y=\int_{y_{2}^{E}}^{\infty} \xi^{E}(y \mid \underline{x}) d y=\frac{\eta}{2} .
$$

Using (6.1), we get (after simplification)

$$
\begin{gathered}
y_{1}^{E}=F^{-1}\left[e^{T\left\{1-(1-(\eta / 2))^{-1 /\left(n-2 c_{1}+1\right)}\right\}}\right] \\
y_{2}^{E}=F^{-1}\left[e^{T\left\{1-(\eta / 2)^{-1 /\left(n-2 c_{1}+1\right)}\right\}}\right]
\end{gathered}
$$

The posterior predictive distribution for $y=x_{n+1}$ given $\underline{x}=\left(x_{1}, x_{2}, \ldots, x_{n}\right)$ under (3.4) is defined by

$$
\begin{aligned}
\xi^{c}(y \mid \underline{x}) & =\int_{0}^{\infty} \pi_{2}(\alpha \mid \underline{x}) g(y ; \alpha) d \alpha \\
& =\frac{n+b}{T_{a}} \frac{1}{\left[1-\left(\ln F(y) / T_{a}\right)\right]^{n+b+1}} \frac{f(y)}{F(y)} .
\end{aligned}
$$

A $100(1-\eta) \%$ equal-tail prediction interval $\left[y_{1}^{c}, y_{2}^{c}\right]$ is the solution of

$$
\int_{0}^{y_{1}^{c}} \xi^{c}(y \mid \underline{x}) d y=\int_{y_{2}^{c}}^{\infty} \xi^{c}(y \mid \underline{x}) d y=\frac{\eta}{2} .
$$

Using (6.4), we get (after simplification)

$$
\begin{gathered}
y_{1}^{c}=F^{-1}\left[e^{T_{a}\left\{1-(1-(\eta / 2))^{-1 /(n+b)}\right\}}\right], \\
y_{2}^{c}=F^{-1}\left[e^{T_{a}\left\{1-(\eta / 2)^{-1 /(n+b)}\right\}}\right] .
\end{gathered}
$$


If $a$ and $b$ are not known, then substituting the empirical Bayes estimate of $a$ and $b$, we get the prediction limits as follows:

$$
\begin{gathered}
y_{1}^{c}=F^{-1}\left[e^{2 T\left\{1-(1-(\eta / 2))^{-1 /(2 n+1)}\right\}}\right] \\
y_{2}^{c}=F^{-1}\left[e^{2 T\left\{1-(\eta / 2)^{-1 / 2 n+1}\right\}}\right] .
\end{gathered}
$$

For deriving classical intervals, we notice that $Z=-\ln F(y) / T$ is distributed as a beta-variate of the second kind with parameters 1 and $n$. The pdf of $Z$ has the following form:

$$
h(z)=\frac{1}{B(1, n)} \frac{1}{(1+z)^{n+1}}, \quad z>0 .
$$

Solving for $\left(z_{1}, z_{2}\right)$ in

$$
\int_{0}^{z_{1}} h(z) d z=\int_{z_{2}}^{\infty} h(z) d z=\frac{\eta}{2}
$$

and using (6.8), we get (after simplification)

$$
\begin{gathered}
z_{1}=F^{-1}\left[e^{T\left\{1-(1-(\eta / 2))^{-1 / n}\right\}}\right], \\
z_{2}=F^{-1}\left[e^{T\left\{1-(\eta / 2)^{-1 / n}\right\}}\right] .
\end{gathered}
$$

It is to be noted that if we take $c_{1}=0.5$ in (6.3), we get classical $100(1-\eta) \%$ equal-tail prediction interval.

\section{Bayes Predictive Estimator and HPD Prediction Interval for a Future Observation}

In this section, we introduce the Bayes predictive estimator for different priors under the above-mentioned loss functions, and later we obtain HPD predictive intervals for the future observation. The Bayes predictive estimators of $y$ under a weighted quadratic error loss function assuming the extended Jeffreys' prior and the conjugate prior are

$$
\begin{aligned}
& y_{1}^{* E}=\frac{\int_{0}^{\infty} y \cdot\left(1 / y^{2}\right) \xi^{E}(y \mid \underline{x}) d y}{\int_{0}^{\infty}\left(1 / y^{2}\right) \xi^{E}(y \mid \underline{x}) d y}, \\
& y_{1}^{* c}=\frac{\int_{0}^{\infty} y \cdot\left(1 / y^{2}\right) \xi^{c}(y \mid \underline{x}) d y}{\int_{0}^{\infty}\left(1 / y^{2}\right) \xi^{c}(y \mid \underline{x}) d y},
\end{aligned}
$$


respectively. The Bayes predictive estimators of $y$ under the squared-log error loss function assuming the extended Jeffreys' prior and the conjugate prior are

$$
\begin{aligned}
& y_{2}^{* E}=\exp [E(\ln Y \mid \underline{x})], \quad \text { with } E(\ln Y \mid \underline{x})=\int_{0}^{\infty} \ln y \cdot \xi^{E}(y \mid \underline{x}) d y, \\
& y_{2}^{* c}=\exp [E(\ln Y \mid \underline{x})], \quad \text { with } E(\ln Y \mid \underline{x})=\int_{0}^{\infty} \ln y \cdot \xi^{c}(y \mid \underline{x}) d y,
\end{aligned}
$$

respectively. The Bayes predictive estimators of $y$ under the general entropy loss function assuming the extended Jeffreys' prior and the conjugate prior are

$$
\begin{gathered}
y_{3}^{* E}=\left[J_{g}^{E}\right]^{-1 / \gamma} \text { with } J_{g}^{E}=\int_{0}^{\infty} y^{-\gamma} \xi^{E}(y \mid \underline{x}) d y, \\
y_{3}^{* c}=\left[J_{g}^{c}\right]^{-1 / \gamma} \text { with } J_{g}^{c}=\int_{0}^{\infty} y^{-\gamma} \xi^{c}(y \mid \underline{x}) d y,
\end{gathered}
$$

respectively. The closed form expressions of (7.1)-(7.6) seem to be intractable, and calculations are to be made using numerical method.

For the unimodal predictive density (6.1), the HPD-predictive interval $\left[h_{1}^{E}, h_{2}^{E}\right]$ with probability $1-\eta$ for $y$ is the simultaneous solution of the following:

$$
P\left(h_{1}^{E}<Y<h_{2}^{E}\right)=1-\eta
$$

that is

$$
\begin{gathered}
\frac{1}{\left[1-\left(\ln F\left(h_{2}^{E}\right) / T\right)\right]^{n-2 c_{1}+1}}-\frac{1}{\left[1-\left(\ln F\left(h_{1}^{E}\right) / T\right)\right]^{n-2 c_{1}+1}}=1-\eta, \\
\xi^{E}\left(h_{1}^{E} \mid \underline{x}\right)=\xi^{E}\left(h_{2}^{E} \mid \underline{x}\right),
\end{gathered}
$$

that is

$$
\left[\frac{1-\left(\ln F\left(h_{2}^{E}\right) / T\right)}{1-\left(\ln F\left(h_{1}^{E}\right) / T\right)}\right]^{n-2 c_{1}+2}=\frac{f\left(h_{2}^{E}\right)}{F\left(h_{2}^{E}\right)} \cdot \frac{F\left(h_{1}^{E}\right)}{f\left(h_{1}^{E}\right)} .
$$

Similarly, for the unimodal predictive density (6.4), the HPD-predictive interval $\left[h_{1}^{c}, h_{2}^{c}\right]$ with probability $1-\eta$ for $y$ is the simultaneous solution of the following:

$$
P\left(h_{1}^{c}<Y<h_{2}^{c}\right)=1-\eta,
$$


that is

$$
\begin{gathered}
\frac{1}{\left[1-\left(\ln F\left(h_{2}^{c}\right) / T_{a}\right)\right]^{n+b}}-\frac{1}{\left[1-\left(\ln F\left(h_{1}^{c}\right) / T_{a}\right)\right]^{n+b}}=1-\eta, \\
\xi^{c}\left(h_{1}^{c} \mid \underline{x}\right)=\xi^{c}\left(h_{2}^{c} \mid \underline{x}\right),
\end{gathered}
$$

that is

$$
\left[\frac{1-\left(\ln F\left(h_{2}^{c}\right) / T_{a}\right)}{1-\left(\ln F\left(h_{1}^{c}\right) / T_{a}\right)}\right]^{n+b+1}=\frac{f\left(h_{2}^{c}\right)}{F\left(h_{2}^{c}\right)} \cdot \frac{F\left(h_{1}^{c}\right)}{f\left(h_{1}^{c}\right)} .
$$

If $a$ and $b$ are not known, then substituting the empirical Bayes estimate of $a$ and $b$, we get the HPD-prediction limits for future observation as follows:

$$
\begin{gathered}
\frac{1}{\left[1-\left(\ln F\left(h_{2}^{c}\right) / 2 T\right)\right]^{2 n+1}}-\frac{1}{\left[1-\left(\ln F\left(h_{1}^{c}\right) / 2 T\right)\right]^{2 n+1}}=1-\eta, \\
{\left[\frac{1-\left(\ln F\left(h_{2}^{c}\right) / 2 T\right)}{1-\left(\ln F\left(h_{1}^{c}\right) / 2 T\right)}\right]^{2 n+2}=\frac{f\left(h_{2}^{c}\right)}{F\left(h_{2}^{c}\right)} \cdot \frac{F\left(h_{1}^{c}\right)}{f\left(h_{1}^{c}\right)} .}
\end{gathered}
$$

\section{Data Analysis}

Consider the following data which arose in tests on endurance of deep grove ball bearings (Lawless [22, page 228]). The data are the number of million revolutions before failure for each of the 23 ball bearings in the life test:

$17.88,28.92,33.00,41.52,42.12,45.60,48.80,51.84,51.96,54.12,55.56,67.80$,

$68.64,68.64,68.88,84.12,93.12,98.64,105.12,105.84,127.92,128.04,173.40$.

To study the goodness of fit of the exponentiated exponential model, Gupta and Kundu [2] computed the $x^{2}$ statistic as 0.783 with the corresponding $P$ value being 0.376 . The estimate of $\alpha$, the shape parameter, is 5.2589 and that of $\theta$, the rate of the exponential distribution, is 0.0314 . Here our aim is to obtain the Bayes estimates of $\alpha$ for this data set under three different loss functions and for two priors by assuming that the base line distribution is exponential with $\theta=0.0314$. At the same time, we are interested in studying the HPD intervals for the parameter $\alpha$. Further, our intention is to obtain the future observation based on a given set of observations and the HPD predictive intervals for the future observation based on the current observations. Figure 6 shows the estimated predictive distribution.

Tables 1-4 summarize the result from the data analysis. Tables $1(\mathrm{a})$ and $1(\mathrm{~b})$ represents the Bayes estimates of $\alpha$ and corresponding risks under the extended Jeffreys' prior the conjugate prior, respectively. It is evident from Table 1(a) that Bayes estimates under general entropy loss $(\gamma=0.5$ and $\gamma=1)$ give better estimates than all other estimates. It is also evident from Table 1(a) that the estimates decrease and the corresponding risks increase with the increase in $c_{1}$. In case of conjugate prior (Table 1(b)), the estimates under weighted 


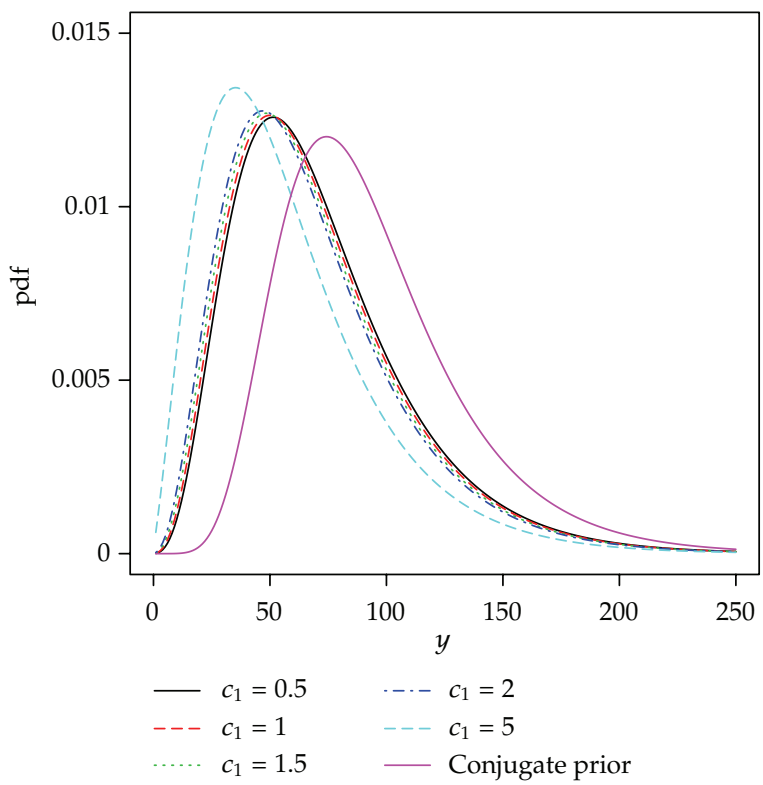

Figure 6: Estimate of the predictive distribution for the given data set for extended Jeffreys' prior and conjugate prior.

Table 1

(a) The Bayes estimates of the shape parameter under extended prior and the corresponding risk function under three different loss functions for the data set

\begin{tabular}{ccccccccccc}
\hline \multirow{2}{*}{$c_{1}$} & \multirow{2}{*}{$\tilde{\alpha}_{\text {bq }}^{E}$} & $R\left(\tilde{\alpha}_{\text {bq }}^{E}\right)$ & \multirow{2}{*}{$\tilde{\alpha}_{\text {bsl }}^{E}$} & $R\left(\tilde{\alpha}_{\text {bsl }}^{E}\right)$ & $\tilde{\alpha}_{\text {bge }}^{E}$ & $R\left(\tilde{\alpha}_{\text {bge }}^{E}\right)$ & $\tilde{\alpha}_{\text {bge }}^{E}$ & $R\left(\tilde{\alpha}_{\text {bge }}^{E}\right)$ & $\tilde{\alpha}_{\text {bge }}^{E}$ & $R\left(\tilde{\alpha}_{\text {bge }}^{E}\right)$ \\
\hline 0.5 & 4.607 & 0.0454 & 4.936 & 0.0444 & 4.881 & 0.0055 & 4.826 & 0.0225 & 4.771 & 0.0511 \\
1.0 & 4.387 & 0.0476 & 4.717 & 0.0465 & 4.662 & 0.0058 & 4.607 & 0.0236 & 4.551 & 0.0535 \\
1.5 & 4.168 & 0.0541 & 4.498 & 0.0531 & 4.443 & 0.0066 & 4.387 & 0.0269 & 4.332 & 0.0611 \\
2.0 & 3.949 & 0.0649 & 4.278 & 0.0649 & 4.223 & 0.0081 & 4.168 & 0.0327 & 4.113 & 0.0741 \\
5.0 & 2.632 & 0.2207 & 2.962 & 0.3052 & 2.907 & 0.0364 & 2.852 & 0.1395 & 2.796 & 0.3012 \\
\hline
\end{tabular}

(b) The Bayes estimates of the shape parameter under conjugate prior and the corresponding risk function under three different loss functions for the data set

\begin{tabular}{|c|c|c|c|c|c|c|c|c|c|}
\hline \multirow{2}{*}{$\tilde{\alpha}_{\mathrm{bq}}^{c}$} & \multirow{2}{*}{$R\left(\widetilde{\alpha}_{\mathrm{bq}}^{c}\right)$} & \multirow{2}{*}{$\tilde{\alpha}_{\mathrm{bsl}}^{c}$} & \multirow{2}{*}{$R\left(\tilde{\alpha}_{\mathrm{bsl}}^{c}\right)$} & \multicolumn{2}{|c|}{$\gamma=0.5$} & \multicolumn{2}{|c|}{$\gamma=1.0$} & \multicolumn{2}{|c|}{$\gamma=1.5$} \\
\hline & & & & $\tilde{\alpha}_{\text {bge }}^{c}$ & $R\left(\tilde{\alpha}_{\mathrm{bge}}^{c}\right)$ & $\tilde{\alpha}_{\text {bge }}^{c}$ & $R\left(\tilde{\alpha}_{\text {bge }}^{c}\right)$ & $\tilde{\alpha}_{\text {bge }}^{c}$ & $R\left(\tilde{\alpha}_{\text {bge }}^{c}\right)$ \\
\hline 4.936 & 0.0503 & 5.101 & 0.0455 & 5.073 & 0.4281 & 5.046 & 1.0691 & 5.018 & 2.0264 \\
\hline
\end{tabular}

quadratic and squared-log error loss functions seem to be better. In Table 2, the HPD intervals under conjugate prior appear to be slightly better than the extended Jeffreys' with respect to minimum length. Table 3 presents the estimates of future observation based on the data set. It is observed from the last column of the table that the general entropy loss function at $r=1.5$ gives a quite reasonable estimate. Table 4 shows the Bayesian predictive and HPD predictive intervals for future observation of the data set. The first row in each cell represents the Bayesian predictive intervals, and the second row represents the HPD intervals for future 
Table 2: HPD intervals of the shape parameter for the data set.

\begin{tabular}{lccc}
\hline$c_{1}$ & & $\left(H_{1}^{E}, H_{2}^{E}\right)$ & $\eta=0.10$ \\
\hline 0.5 & $(2.631,7.997)$ & $\eta=0.05$ & $(3.019,6.531)$ \\
1.0 & $(2.472,7.718)$ & $(2.999,7.079)$ & $(2.999,6.361)$ \\
1.5 & $(2.314,7.437)$ & $(2.902,6.878)$ & $(2.961,6.214)$ \\
2.0 & $(2.158,7.154)$ & $(2.558,6.612)$ & $(2.782,5.954)$ \\
5.0 & $(2.061,7.052)$ & $(2.365,6.131)$ & $(2.532,5.691)$ \\
$\left(H_{1}^{c}, H_{2}^{c}\right)$ & $(2.108,7.065)$ & $(2.236,6.452)$ & $(2.871,6.141)$ \\
\hline
\end{tabular}

Table 3: Estimated future observation for the data set under three different loss functions.

\begin{tabular}{lccccc}
\hline$c_{1}$ & $y_{1}^{*}$ & $y_{2}^{*}$ & $\gamma=0.5$ & $\gamma=1.0$ & $\gamma=1.5$ \\
\hline 0.5 & 33.359 & 62.788 & 57.977 & 53.098 & 48.026 \\
1.0 & 30.572 & 61.338 & 56.432 & 51.431 & 46.169 \\
1.5 & 26.726 & 59.822 & 54.812 & 49.673 & 44.173 \\
2.0 & 24.755 & 58.233 & 53.111 & 47.813 & 42.017 \\
5.0 & 18.285 & 46.701 & 40.504 & 33.421 & 27.315 \\
conjugate prior & 55.329 & 13.047 & 320.483 & 315.724 & 190.144 \\
\hline
\end{tabular}

Table 4: Bayesian predictive and HPD predictive intervals for future observation of the data set.

\begin{tabular}{ccccccc}
\hline$\eta$ & Conjugate prior & $c_{1}=0.5$ & $c_{1}=1.0$ & $c_{1}=1.5$ & $c_{1}=2.0$ & $c_{1}=5.0$ \\
\hline \multirow{2}{*}{0.01} & $(0.015,17.32)$ & $(0.031,37.61)$ & $(0.033,39.53)$ & $(0.034,41.66)$ & $(0.036,44.03)$ & $(0.051,66.78)$ \\
& $(7.819,220.83)$ & $(5.966,199.82)$ & $(5.257,198.29)$ & $(4.552,196.67)$ & $(3.856,194.97)$ & $(0.398,182.53)$ \\
\hline \multirow{2}{*}{0.05} & $(0.078,11.85)$ & $(0.159,25.25)$ & $(0.167,26.49)$ & $(0.175,27.87)$ & $(0.183,29.41)$ & $(0.262,43.76)$ \\
& $(16.206,168.96)$ & $(10.712,148.26)$ & $(10.217,147.45)$ & $(9.654,146.41)$ & $(8.671,144.69)$ & $(2.635,131.90)$ \\
\hline \multirow{2}{*}{0.10} & $(0.158,9.55)$ & $(0.324,20.19)$ & $(0.338,21.17)$ & $(0.354,22.25)$ & $(0.372,23.45)$ & $(0.532,34.63)$ \\
& $(26.138,146.11)$ & $(14.176,124.48)$ & $(13.812,123.37)$ & $(12.103,122.28)$ & $(10.916,121.24)$ & $(4.939,110.22)$ \\
\hline
\end{tabular}

observation. It is apparent that the HPD intervals for future observation with respect to conjugate prior are reasonably good.

\section{Concluding Remark}

In this paper, we have derived the Bayes estimators of the shape parameter of the exponentiated family of distributions under the extended Jeffreys' prior as well as conjugate prior using three different loss functions. Though the extended Jeffreys' prior gives the opportunity of covering wide spectrum of priors, yet at times the conjugate prior also gives better Bayes estimates and HPD intervals of the parameter and of future observations.

\section{Acknowledgment}

The authors would like to thank the referee for a very careful reading of the manuscript and making a number of nice suggestions which improved the earlier version of the manuscript. 


\section{References}

[1] R. D. Gupta and D. Kundu, "Generalized exponential distributions," Australian and New Zealand Journal of Statistics, vol. 41, no. 2, pp. 173-188, 1999.

[2] R. D. Gupta and D. Kundu, "Exponentiated exponential family: an alternative to gamma and Weibull distributions," Biometrical Journal, vol. 43, no. 1, pp. 117-130, 2001.

[3] R. D. Gupta and D. Kundu, "Generalized exponential distribution: different method of estimations," Journal of Statistical Computation and Simulation, vol. 69, no. 4, pp. 315-337, 2001.

[4] R. D. Gupta and D. Kundu, "Discriminating between Weibull and generalized exponential distributions," Computational Statistics E Data Analysis, vol. 43, no. 2, pp. 179-196, 2003.

[5] M. Z. Raqab, "Inferences for generalized exponential distribution based on record statistics," Journal of Statistical Planning and Inference, vol. 104, no. 2, pp. 339-350, 2002.

[6] M. Z. Raqab and M. Ahsanullah, "Estimation of the location and scale parameters of generalized exponential distribution based on order statistics," Journal of Statistical Computation and Simulation, vol. 69, no. 2, pp. 109-123, 2001.

[7] G. Zheng, "On the Fisher information matrix in type II censored data from the exponentiated exponential family," Biometrical Journal, vol. 44, no. 3, pp. 353-357, 2002.

[8] M. Z. Raqab and M. T. Madi, "Bayesian inference for the generalized exponential distribution," Journal of Statistical Computation and Simulation, vol. 75, no. 10, pp. 841-852, 2005.

[9] A. A. Alamm, M. Z. Raqab, and M. T. Madi, "Bayesian prediction intervals for future order statistics from the generalized exponential distribution," Journal of the Iranian Statistical Society, vol. 6, no. 1, pp. 17-30, 2007.

[10] R. Singh, S. K. Singh, U. Singh, and G. P. Singh, "Bayes estimator of generalized-exponential parameters under Linex loss function using Lindley's approximation," Data Science Journal, vol. 7, pp. 65-75, 2008.

[11] S. Dey, "Bayesian estimation of the shape parameter of the generalised exponential distribution under different loss functions," Pakistan Journal of Statistics and Operations Research, vol. 6, no. 2, pp. 163-174, 2010.

[12] G. S. Mudholkar and D. K. Srivastava, "Exponentiated Weibull family for analyzing bathtub failurerate data," IEEE Transactions on Reliability, vol. 42, no. 2, pp. 299-302, 1993.

[13] M. M. Nassar and F. H. Eissa, "Bayesian estimation for the exponentiated Weibull model," Communications in Statistics. Theory and Methods, vol. 33, no. 10, pp. 2343-2362, 2004.

[14] U. Singh, P. K. Gupta, and S. K. Upadhyay, "Estimation of parameters for exponentiated-Weibull family under type-II censoring scheme," Computational Statistics and Data Analysis, vol. 15, pp. 20652077, 2005.

[15] R. D. Gupta and R. C. Gupta, “Analyzing skewed data by power normal model,” TEST, vol. 17, no. 1, pp. 197-210, 2008.

[16] H. S. Al-Kutubi, On comparison estimation procedures for parameter and survival function exponential distribution using simulation, Ph.D. thesis, Baghdad University, Baghdad, Iraq, 2005.

[17] L. Brown, "Inadmissiblity of the usual estimators of scale parameters in problems with unknown location and scale parameters," Annals of Mathematical Statistics, vol. 39, pp. 29-48, 1968.

[18] R. Calabria and G. Pulcini, "Point estimation under asymmetric loss functions for left-truncated exponential samples," Communications in Statistics. Theory and Methods, vol. 25, no. 3, pp. 585-600, 1996.

[19] C. K. Podder and M. K. Roy, "Bayesian estimation of the parameter of Maxwell distribution under MLINEX loss function," Journal of Statistical Studies, vol. 23, pp. 11-16, 2003.

[20] D. V. Lindley, Introduction to Probability and Statistics from a Bayesian View Point, Cambridge University Press, Cambridge, Mass, USA, 1969.

[21] A. M. Awad and M. K. Gharraf, "Estimation of $P[Y<X]$ in the Burr case: a comparative study," Communications in Statistics-Simulation and Computation, vol. 15, no. 2, pp. 389-403, 1986.

[22] J. F. Lawless, Statistical Models and Methods for Lifetime Data, John Wiley and Sons, New York, NY, USA, 1982. 


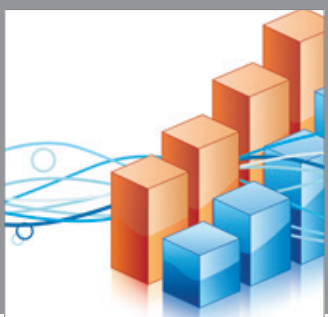

Advances in

Operations Research

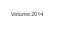

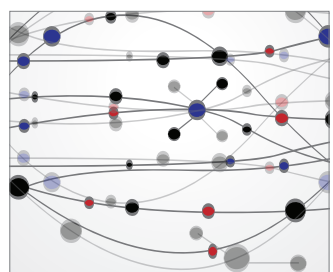

\section{The Scientific} World Journal
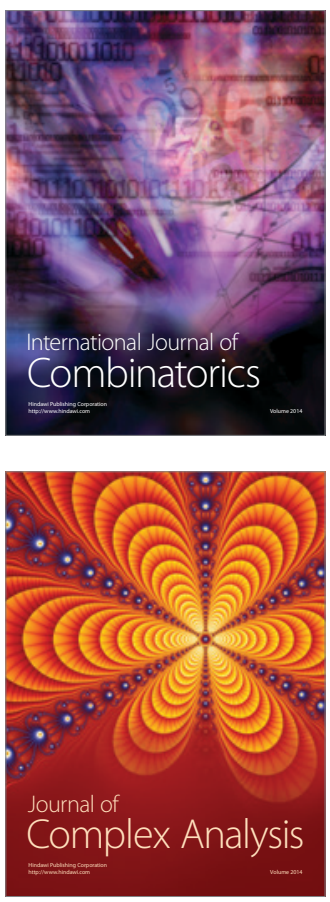

International Journal of

Mathematics and

Mathematical

Sciences
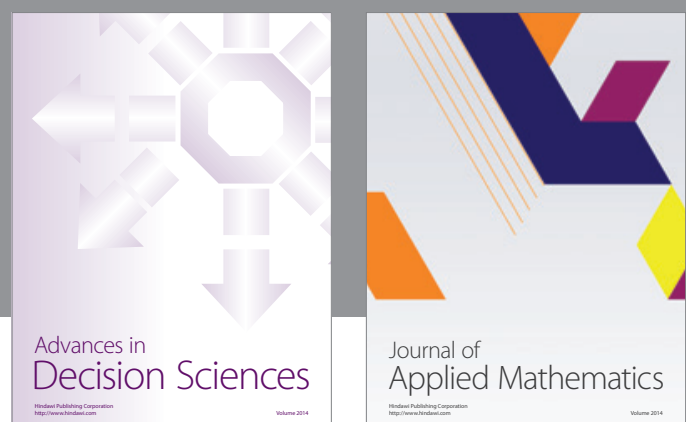

Journal of

Applied Mathematics
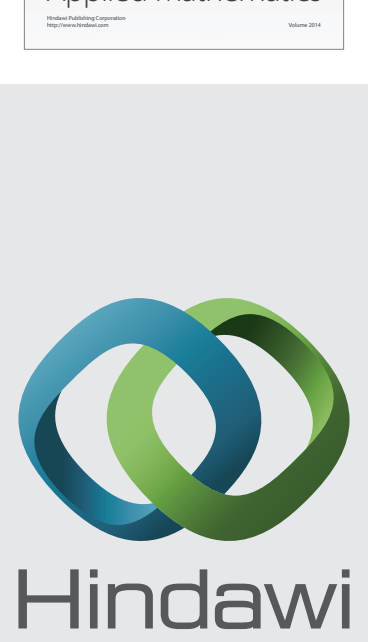

Submit your manuscripts at http://www.hindawi.com
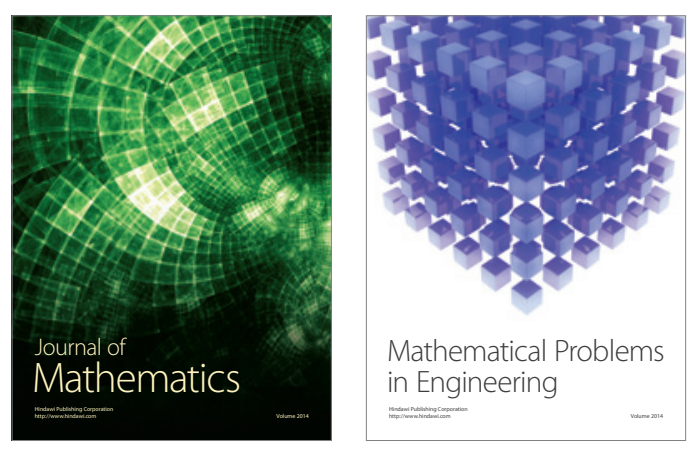

Mathematical Problems in Engineering
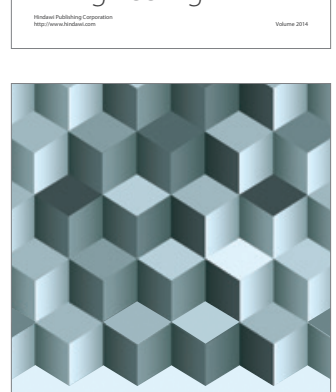

Journal of

Function Spaces
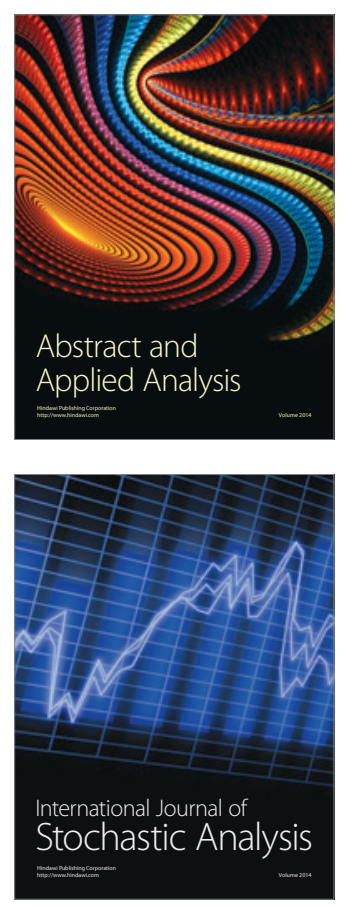

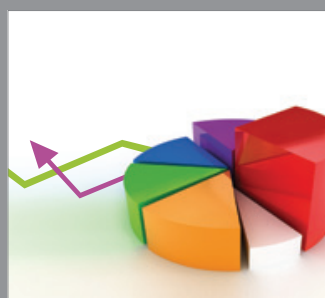

ournal of

Probability and Statistics

Promensencen
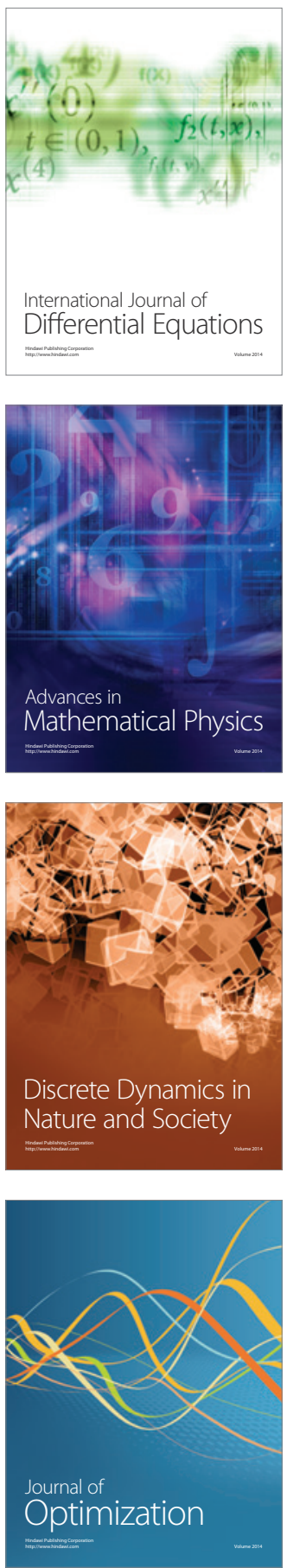